\title{
A systematic review of randomized controlled trials on exercise parameters in the treatment of patellofemoral pain: what works?
}

This article was published in the following Dove Press journal:

Journal of Multidisciplinary Healthcare

28 October 20II

Number of times this article has been viewed

\author{
Daniel Harvie \\ Timothy O'Leary \\ Saravana Kumar \\ International Centre for Allied \\ Health Evidence (iCAHE), City East \\ Campus, University of South Australia, \\ Adelaide, Australia
}

Purpose: There is research evidence which supports the effectiveness of exercise in reducing pain and increasing function in patients with patellofemoral pain syndrome. However, what is unclear are the parameters underpinning this intervention. This has led to uncertainty when operationalizing exercises for patients with patellofemoral pain syndrome in clinical practice. The aim of this review was to evaluate the parameters of exercise programs reported in primary research, to provide clinicians with evidence-based recommendations for exercise prescription for patellofemoral pain.

Methods: A systematic review of randomized controlled trials was undertaken. Only trials that identified exercise to be effective in treating patellofemoral pain were included. Appropriate databases and reference lists were searched using established keywords. Data relating to common exercise parameters such as the type of exercise, length, and frequency of intervention, intensity, repetitions, sets, and specific technique were extracted, along with details of co-interventions that may have been used.

Results: A total of ten randomized controlled trials were included in this review and from these trials 14 interventions arms were evaluated. All 14 interventions focused on active exercises, all but two of which also included a passive stretching component. The current body of evidence demonstrates positive results with exercise interventions such as knee extension, squats, stationary cycling, static quadriceps, active straight leg raise, leg press, and step-up and down exercises for patients with patellofemoral pain syndrome. A progressive regime of daily exercises of two to four sets of ten or more repetitions over an intervention period of 6 weeks or more, combined with exercises to address flexibility of the lower limb musculature was commonly used.

Conclusion: Currently, the primary research on this topic supports the use of closed kinetic chain, strengthening exercises for musculature of the lower limb, combined with flexibility options. The current evidence base supports a prescription of daily exercises of two-four sets of ten or more repetitions over a period of 6 weeks or more.

Keywords: patellofemoral pain syndrome, PFPS, repetitions, lower limb, musculature

\section{Introduction}

Patellofemoral pain syndrome (PFPS) is one of the most common knee conditions seen by physiotherapists, affecting one in four people of the total population. ${ }^{1}$ PFPS is characterized as a diffuse retro/peripatellar pain, aggravated with activities which load the patellofemoral joint, such as climbing stairs, squatting, running, and prolonged sitting. ${ }^{2}$ Although its precise etiology is not currently universally accepted, ${ }^{2}$ several neuromuscular deficits have been associated with its development, forming the basis for a plethora of different treatment options employed by researchers and clinicians alike. ${ }^{3}$ Conservative management remains the treatment of choice
Correspondence: Saravana Kumar International Centre for Allied Health Evidence (iCAHE), University of South Australia, City East Campus, GPO Box 247I, Adelaide 5000, Australia

Tel +6I 883022085

Fax +6I 883022766

Email saravana.kumar@unisa.edu.au 
for frontline management of PFPS, with exercise therapy forming the mainstay. ${ }^{4}$ These treatments target neuromuscular deficits, including quadriceps, vastus medialis oblique (VMO), proximal strength deficits, tightness of soft tissues, or dynamic alignment/control abnormalities identified by the treating clinician. ${ }^{5}$ The range of exercises employed to target these deficits include various combinations and variations of open and closed kinetic chain exercises, exercises aimed at selectively or nonselectively recruiting muscles, and stretching. ${ }^{3}$

Systematic reviews on this topic have focused on the question of whether exercise is effective for PFPS and have reported mixed findings. Heintjes et $\mathrm{al}^{6}$ reported conflicting results; however, a more recent review by Fagan and Delahunt $^{3}$ revealed that some exercise interventions have been shown to reduce pain and increase function in PFPS patients. Since then several randomized controlled trials have demonstrated positive results in pain and function using exercise-based interventions..$^{7-11}$

The plethora of different exercise interventions reported in the literature suggests a lack of universally accepted exercise protocols for the management of PFPS. The aim of this systematic review was to analyze the exercise parameters associated with positive, statistically significant effects on PFPS, as reported in the primary research, in order to advise clinicians on the evidence-based recommendations for the treatment of patellofemoral pain.

\section{Methods}

\section{Search strategy}

Figure 1 provides a CONSORT (Consolidated Standards of Reporting Trials) diagram summary of the search strategy employed.

\section{Databases}

The following databases were searched using the devised PICO (patient, intervention, comparison, outcome) (Table 1) between January 13 and February 17, 2010: SPORTDiscus, MEDLINE, CINAHL, Science Direct, PEDro, EMBASE, and Google Scholar. The search terms used were: "patellofemoral pain syndrome" OR "patellofemoral pain" OR "retropatellar pain" AND “exercise” OR "exercise therapy" OR "strength" OR "rehabilitation." The following limits were placed on the search where able: English language, humans, age 14-65, randomized controlled trials, and years 1996-2010. The reference lists of trials identified from the databases were manually searched for potentially relevant trials.

\section{Inclusion}

All randomized controlled trials from peer reviewed journals written in English, available in full text, and matching the above selection criteria were included in order to increase the rigor of the systematic review design. To address this study's research question, only trials which demonstrated a positive outcome due to the intervention were included. This allowed analysis of the parameters of exercise, which led to positive outcomes in trials thus addressing the review aims. A positive outcome is defined as "a statistically significant improvement in one or more measure of pain and function." Exercise interventions with nonsignificant outcomes when compared with other treatments were included if at least one arm showed significant improvement from baseline scores, irrespective of whether there was a significant difference between intervention groups.

\section{Exclusion}

A trial was excluded from this review if the trial's primary intervention was a non-exercise based intervention, or the trial did not include at least one measure of pain and function.

\section{Methodological assessment}

All included trials were critically appraised by two independent reviewers using the PEDro scale, ${ }^{12}$ an eleven-point scale commonly used to rate the methodology of randomized controlled trials. The inter-rater reliability of the PEDro score has previously been shown to be "fair to good." mentary material for the PEDro critical appraisal criteria.

\section{Data extraction}

Relevant data was extracted manually and entered into the data extraction table (see Table 2). The parameters used were: type of exercise (eg, open or closed chain), length of intervention, frequency, sets, repetitions of exercise, intensity (eg, $60 \%$ one-repetition maximum [1RM]), co-interventions (eg, taping), and instructions/specific technique where relevant (eg, alignment or avoidance of pain during exercise).

\section{Results \\ Search findings}

The literature search revealed ten studies meeting the systematic review criteria. Fourteen intervention arms reporting statistically significant improvements were included. All included trials were from peer reviewed journals. One study ${ }^{14}$ was excluded due to its poor methodological quality and high degree of bias. 


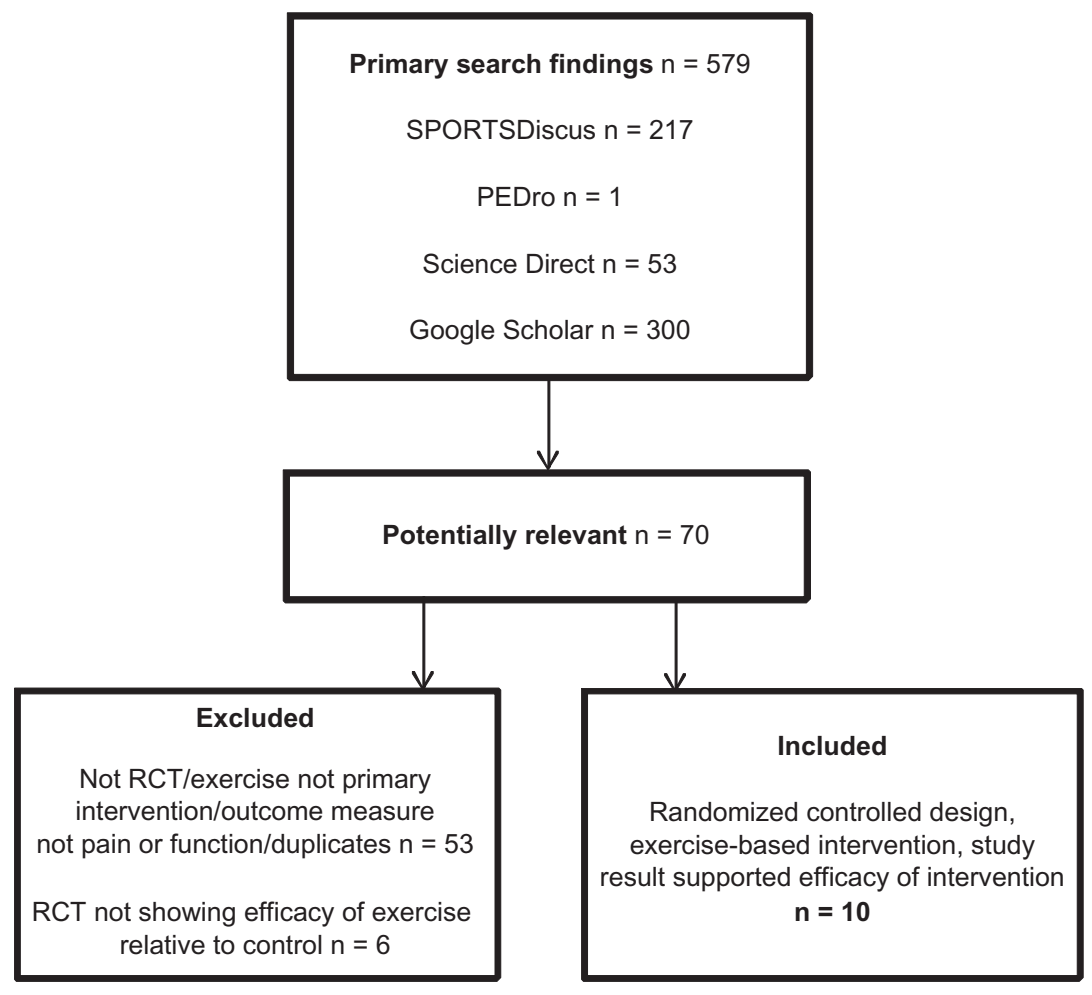

Figure I CONSORT (Consolidated Standards of Reporting Trials) diagram summarizing search and selection process. Abbreviation: RCT, randomized controlled trial.

\section{Quality of the studies included}

Studies meeting the inclusion criteria were scored using the PEDro critical appraisal tool. Within an exercise intervention protocol it is not possible to blind either therapist or participant, leaving the maximum score possible as 9 out of 11 . The included studies scored in the range of 6-9 on the PEDro scale (see Figure 2).

\section{The interventions}

Table 2 details the exercise parameters used in each of the studies. It shows the type of exercise done, the frequency, duration, intensity, and number of repetitions/sets that were performed.

\section{Exercise type}

The common interventions can be broadly categorized as "open" or "closed" kinetic chain and "selective" or "nonselective"

Table I The search PICO used to develop keywords

\begin{tabular}{ll}
\hline Population & $\begin{array}{l}\text { Aged 14-65 years of either gender clinically } \\
\text { diagnosed with PFJ pain }\end{array}$ \\
Intervention & $\begin{array}{l}\text { Exercise-based rehabilitation for PFJ } \\
\text { Other interventions including placebo/other exercise/ } \\
\text { Comparison }\end{array}$ \\
nonexercise-based therapy \\
Outcome & Psychometrically sound measure of pain and function \\
\hline
\end{tabular}

Abbreviation: PFJ, patellofemoral joint. exercises. Open chain exercises were used in eight programs, while closed chain exercises were employed by eleven. Stretching was a feature of all but one protocol and was done concurrently with the strengthening program. "Proprioceptive/ balance" exercise type ${ }^{11,15}$ and "proximal stability" were features of individual studies. ${ }^{19}$ Exercises were designed primarily to target strength and/or control at the knee or hip, and to correct local or peripheral muscle imbalance.

\section{Program duration and frequency}

All trials reported frequency per week and duration in weeks. The most common frequency of exercise was a 6-week period of daily exercise employed by four of the ten included trials. ${ }^{8,9,15,16}$ Three of the included studies employed 8-week rehabilitation periods, ${ }^{10}$ with the remaining three studies employing 3-week, ${ }^{7}$ 5-week, ${ }^{4}$ and 12-week ${ }^{11}$ rehabilitation periods respectively. Although Bakhtiary and Fatemi ${ }^{7}$ had the shortest intervention period of 3 weeks, the frequency of exercise sessions were the greatest (twice daily), bringing the total volume of exercise sessions more in line with the remaining literature that reported positive effects of exercise on PFPS.

\section{Specific exercises used}

The exercises included in the randomized controlled trials were open kinetic chain (OKC), closed kinetic chain (CKC), 
Table 2 Characteristics of effective exercise interventions for patellofemoral pain

\begin{tabular}{|c|c|c|c|c|c|c|c|}
\hline \multirow[t]{2}{*}{ Study } & \multicolumn{4}{|l|}{ Intervention } & \multicolumn{3}{|c|}{ Program parameters } \\
\hline & Type of exercise & $\begin{array}{l}\text { Duration } \\
\text { (weeks) }\end{array}$ & Frequency & Exercises & $\begin{array}{l}\text { Intensityl } \\
\text { resistance }\end{array}$ & Reps & Sets \\
\hline \multirow[t]{7}{*}{ Clark et al ${ }^{15}$} & CKC including & 6 & Daily & Stationary cycle (warm-up) & NR & NR & NR \\
\hline & proprioceptive/ & & & Wall squats & & $10 \times 10$-second & I \\
\hline & balance exercises & & & Sit to stand & & holds, & NR \\
\hline & & & & Progressive step down exercise & & progressed & \\
\hline & & & & Specific gluteus medius & & to 3 minutes & \\
\hline & & & & and maximus exercises & & NR & \\
\hline & & & & Balance work using trampet & & & \\
\hline \multirow[t]{10}{*}{ Crossley et al ${ }^{16}$} & CKC, isometric & 6 & Daily & Week I-2: & & & \\
\hline & exercises including & & & Isometric VMO (knee at $90^{\circ}$ ) & NR & 10 & 4 \\
\hline & selective VMO & & & $\begin{array}{l}\text { Squats to } 40^{\circ} \text { knee flexion }+ \\
\text { isometric gluteals }\end{array}$ & & 10 & 4 \\
\hline & & & & Isometric abduction against & & I5-second hold & 4 \\
\hline & & & & a wall in standing & & & \\
\hline & & & & Week 3-6: & & & \\
\hline & & & & Step downs $10-20 \mathrm{~cm}$ depth & & $5-10$ & 3 \\
\hline & & & & Isometric hip abduction & & 30-second hold & 4 \\
\hline & & & & in standing & & & \\
\hline & & & & All week I-2 exercises & & & \\
\hline Nakagawa & OKC and CKC & 6 & $5 \times /$ week & Week I-2: & & & \\
\hline \multirow[t]{36}{*}{ et $\mathrm{al}^{9}$} & $\begin{array}{l}\text { including proximal } \\
\text { stability }\end{array}$ & & & $\begin{array}{l}\text { TrA contractions in } \\
\text { quadruped kneel }\end{array}$ & NR & $\begin{array}{l}15 \times 10 \text {-second } \\
\text { holds }\end{array}$ & 2 \\
\hline & & & & Isometric combined hip & & $15 \times 10$-second & 2 \\
\hline & & & & abduction and lateral rotation & & holds & \\
\hline & & & & in side lying, hips and knees & & & \\
\hline & & & & slightly flexed & & & \\
\hline & & & & Isometric hip abduction with & & $15 \times 10$-second & 2 \\
\hline & & & & knees extended & & holds & \\
\hline & & & & Isometric hip abduction and & & $15 \times 10$-second & 2 \\
\hline & & & & lateral rotation in quadruped & & holds & \\
\hline & & & & Isometric quadriceps & & $15 \times 10$-second & 2 \\
\hline & & & & (knee at $90^{\circ}$ ) & & holds & \\
\hline & & & & ASLR & & 10 & 3 \\
\hline & & & & Squat to $40^{\circ}$ flexion & & 10 & 4 \\
\hline & & & & Week 3-4: & & & \\
\hline & & & & Wall squat $0^{\circ}-60^{\circ}$ flexion & & 10 & 3 \\
\hline & & & & Step up/down $(20 \mathrm{~cm}$ depth $)$ & & 5 & 3 \\
\hline & & & & Lunges $0^{\circ}-45^{\circ}$ flexion & & 10 & 3 \\
\hline & & & & Pelvic drop exercise on & & 15 & 2 \\
\hline & & & & $20 \mathrm{~cm}$ step & & & \\
\hline & & & & Single arm extension against & & 10 & 4 \\
\hline & & & & elastic resistance in & & & \\
\hline & & & & contralateral single leg stance & & & \\
\hline & & & & Contralateral body rotation in & & $15 \times 10$-second & 2 \\
\hline & & & & single leg stance against elastic & & holds & \\
\hline & & & & resistance, maintaining lower & & & \\
\hline & & & & limb static & & & \\
\hline & & & & Weeks 5-6: & & & \\
\hline & & & & As for week 3 and $4+$ balance & & As per week 3-4 & \\
\hline & & & & exercises and additional & & & \\
\hline & & & & elastic resistance around the & & & \\
\hline & & & & affected leg in forward lunges, & & & \\
\hline & & & & encouraging active lateral & & & \\
\hline & & & & rotation and abduction & & & \\
\hline & & & & of the hip. & & & \\
\hline & & & & Begin progress walk or & & & \\
\hline & & & & run program & & & \\
\hline
\end{tabular}


Table 2 (Continued)

\begin{tabular}{|c|c|c|c|c|c|c|c|}
\hline \multirow[t]{2}{*}{ Study } & \multicolumn{4}{|l|}{ Intervention } & \multicolumn{3}{|c|}{ Program parameters } \\
\hline & Type of exercise & $\begin{array}{l}\text { Duration } \\
\text { (weeks) }\end{array}$ & Frequency & Exercises & $\begin{array}{l}\text { Intensity/ } \\
\text { resistance }\end{array}$ & Reps & Sets \\
\hline \multirow[t]{2}{*}{$\begin{array}{l}\text { Bakhtiary and } \\
\text { Fatemi }^{7}\end{array}$} & Group I: OKC & 3 & $2 \times /$ day & $\begin{array}{l}\text { Supine straight leg raise } \\
\text { to } 45^{\circ} \text { hip flexion }\end{array}$ & NR & $\begin{array}{l}\text { Group I and 2: } \\
20 \times 3-4 \text {-second }\end{array}$ & 1 \\
\hline & Group 2: CKC & 3 & $2 \times /$ day & $\begin{array}{l}\text { Single leg squat to } 15^{\circ}-20^{\circ} \\
\text { to knee flexion whilst holding } \\
\text { stable surface }\end{array}$ & & $\begin{array}{l}\text { hold, increased } \\
\text { by } 5 \text { reps every } \\
2 \text { days }\end{array}$ & \\
\hline \multirow[t]{2}{*}{$\begin{array}{l}\text { Witvrouw } \\
\text { et al }{ }^{19}\end{array}$} & Group I: OKC & 5 & $3 \times /$ week & $\begin{array}{l}\text { Static quadriceps contractions } \\
\text { in full knee extension } \\
\text { Straight leg raise in supine } \\
\text { Knee extension from } 10^{\circ} \\
\text { flexion to full extension } \\
\text { Leg adduction exercises } \\
\text { in side lying }\end{array}$ & $\begin{array}{l}\text { Groups I and 2: } \\
60 \% \text { of IORM, new } \\
\text { IORM established } \\
\text { at the end of each } \\
\text { week. "Maximal } \\
\text { contractions" for } \\
\text { static tasks }\end{array}$ & $\begin{array}{l}\text { Group I: } \\
10 \times 6 \text {-second } \\
\text { hold }\end{array}$ & 3 \\
\hline & Group 2: CKC & 5 & $3 \times /$ week & $\begin{array}{l}\text { Seated leg press } \\
\text { Double or single leg squat } \\
\text { I/3 knee bend } \\
\text { Stationary biking } \\
\text { Rowing machine exercise } \\
\text { Step up and down exercise }\end{array}$ & & Group 2: 10 & 3 \\
\hline Herrington and & Group I: OKC & 6 & $3 \times /$ week & $\begin{array}{l}\text { Knee extension exercises in } \\
\text { seated position from } 90^{\circ} \\
\text { of flexion to full extension }\end{array}$ & $\begin{array}{l}\text { Groups I and 2: } \\
\text { Set I 50\% 6RM } \\
\text { Set } 275 \% \text { 6RM }\end{array}$ & $\begin{array}{l}10 \\
10 \\
\text { Max possible }\end{array}$ & 4 \\
\hline Al-Sherhi ${ }^{8}$ & Group 2: CKC & 6 & $3 \times /$ week & $\begin{array}{l}\text { Leg press in seated position } \\
\text { from } 90^{\circ} \text { of knee flexion } \\
\text { to full extension } \\
\text { Group I and } 2 \text { : } 5 \text {-minute } \\
\text { static cycle warm-up }\end{array}$ & $\begin{array}{l}\text { Set } 3100 \% 6 \mathrm{RM} \\
\text { Set } 4 \text { Progressed } \\
\text { according } \\
\text { set } 3 \text { max } \\
\text { (Next session } \\
\text { progressed } \\
\text { based } \\
\text { on set } 4 \text { max) }\end{array}$ & Max possible & \\
\hline \multirow[t]{2}{*}{ Syme et al ${ }^{10}$} & $\begin{array}{l}\text { Group I: General } \\
\text { non-VMO } \\
\text { selective }\end{array}$ & 8 & $2 \times /$ week & $\begin{array}{l}3-5 \text { lower limb exercises } \\
\text { focusing on quadricep } \\
\text { strengthening. Actual } \\
\text { exercises not specified. }\end{array}$ & $\begin{array}{l}60 \%-70 \% \\
\text { of IRM }\end{array}$ & 10 & $1-3$ \\
\hline & $\begin{array}{l}\text { Group 2: VMO } \\
\text { selective }\end{array}$ & 8 & $\begin{array}{l}\text { Daily HEP + } \\
>6 \text { EMG } \\
\text { biofeedback } \\
\text { sessions }\end{array}$ & $\begin{array}{l}\text { Lower limb exercises focusing } \\
\text { on selective activation and } \\
\text { retraining of the VMO } \\
\text { relative to the VL using } \\
\text { a dual channel surface } \\
\text { electromyographic } \\
\text { (EMG) biofeedback unit. } \\
\text { Daily home exercises }\end{array}$ & NR & NR & $N R$ \\
\hline $\begin{array}{l}\text { Van Linschoten } \\
\text { et al"l }\end{array}$ & $\begin{array}{l}\mathrm{OKC} \text { and } \mathrm{CKC} \\
\text { including balance } \\
\text { exercises }\end{array}$ & 12 & $\begin{array}{l}25 \text { minutes } \\
\text { daily }\end{array}$ & $\begin{array}{l}\text { Stationary cycle (warm-up), } \\
\text { static and dynamic } \\
\text { strengthening } \\
\text { exercises for quadriceps, } \\
\text { adductors, and gluteal } \\
\text { muscles and balance } \\
\text { work }\end{array}$ & $\begin{array}{l}\text { NR - though } \\
\text { progressed } \\
\text { fortnightly } \\
\text { during the } \\
\text { first } 6 \text { weeks }\end{array}$ & $\begin{array}{l}\text { NR - though } \\
\text { progressed } \\
\text { fortnightly } \\
\text { during the first } \\
6 \text { weeks }\end{array}$ & NR \\
\hline Song et al ${ }^{17}$ & CKC & 8 & $3 \times /$ week & SL leg press, $45^{\circ}-0^{\circ}$ flexion & $\begin{array}{l}60 \% \text { IRM } \\
\text { (IRM reset } \\
\text { every } 2 \text { weeks) }\end{array}$ & 10 & 5 \\
\hline Kettunen & OKC and CKC & 8 & Daily & Weeks I-4: & NR & Weeks I-2: & \\
\hline \multirow[t]{3}{*}{ et $\mathrm{al}^{23}$} & & & & $\begin{array}{l}\text { Standing hamstring curl } \\
\text { All fours, transverse }\end{array}$ & & $\begin{array}{l}10-20 \\
\text { Weeks 2-4: }\end{array}$ & 2 \\
\hline & & & & plane single & & $10-40$ & 4 \\
\hline & & & & leg bent knee hip abduction & & Weeks 4-6: & \\
\hline
\end{tabular}


Table 2 (Continued)

\begin{tabular}{|c|c|c|c|c|c|c|c|}
\hline \multirow[t]{2}{*}{ Study } & \multicolumn{4}{|l|}{ Intervention } & \multicolumn{3}{|c|}{ Program parameters } \\
\hline & Type of exercise & $\begin{array}{l}\text { Duration } \\
\text { (weeks) }\end{array}$ & Frequency & Exercises & $\begin{array}{l}\text { Intensityl } \\
\text { resistance }\end{array}$ & Rep & Sets \\
\hline & & & & Knee extension & & $10-20$ & 2 \\
\hline & & & & Straight leg raise & & Weeks 6-8: & \\
\hline & & & & Weeks 4-8: & & $10-40$ & 4 \\
\hline & & & & Standing hip extension & & & \\
\hline & & & & against resistance band with & & & \\
\hline & & & & straight knee & & & \\
\hline & & & & Standing hip extension against & & & \\
\hline & & & & resistance band moving from & & & \\
\hline & & & & knee flexion to extension & & & \\
\hline & & & & Lateral step down/up & & & \\
\hline & & & & Sit to stand & & & \\
\hline
\end{tabular}

Abbreviations: reps, repetitions; NR, not reported; CKC, closed kinetic chain; OKC, open kinetic chain; SLR, straight leg raise; ASLR, active straight leg raise; RM, repetition max; TrA, transversus abdominus; VMO, vastus medialis oblique.

selective, stability/proximal control, and general exercises. The specific exercises reported in the trials for open chain exercises were: active straight leg raise $(n=4)$, knee extension $(n=3)$, and static quadriceps $(n=3)$. Closed chain exercises were: double/single leg squats $(n=5)$, step up and step down $(n=5)$, seated leg press $(n=3)$, static quadriceps contraction in knee flexion $(n=2)$, stationary bike $(n=4)$, and static hip abduction $+/-$ external rotation $(\mathrm{n}=2)$, with several other exercises appearing just once (eg, lunges and rowing machine). Selective exercises were selective VMO exercises $(n=2)$. Stability/proximal control exercises included: balance work $(\mathrm{n}=2)$ and proximal stability/transversus abdominus (TrA) $(n=1)$. General exercises included progressive walk/run program $(\mathrm{n}=1)$.

\section{Intensity}

Intensity ranged from varying percentages of maximum effort to failure to report. Whilst there was variability in the prescribed exercise intensities, there was a trend to increase the intensity/resistance progressively throughout the rehabilitation period, with three studies ${ }^{4,8,10}$ reporting this parameter and also

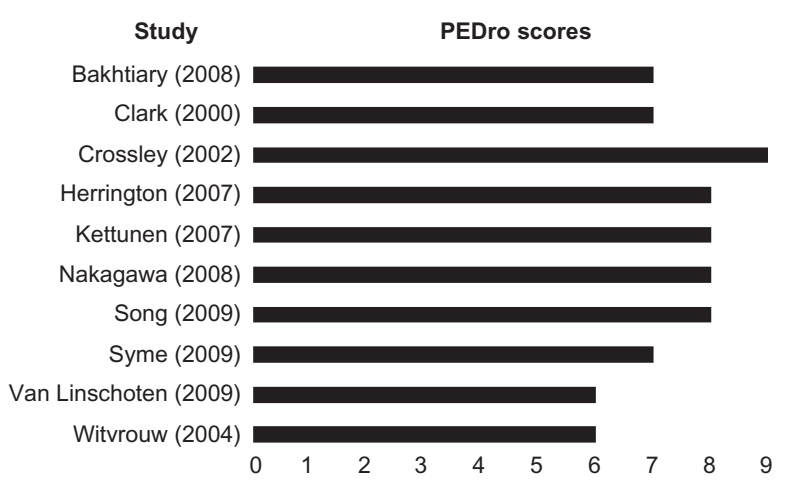

Figure 2 Graph showing PEDro scores for each of the included studies. describing its progression. The lowest frequency of exercise was two times a week but with the greatest intensity (60\%-70\% of 1RM) of all the included studies. ${ }^{10}$ Similar prescription was practiced by three other trials ${ }^{4,8,17}$ with an exercise frequency of three times a week, but with higher intensities of exercise than the remaining groups. Where intensity was given, it was always above $60 \%$ of ten-repetitions maximum (10RM).

\section{Sets and repetitions}

The included trials reported a minimum of ten repetitions, except two studies ${ }^{9,16}$ which involved lengthy isometric contractions repeated a lesser number of times. Studies included between one and four sets, with larger numbers of sets when the repetitions were lower (see Crossley et $\mathrm{al}^{16}$ ) or lower numbers of sets where the repetitions were higher (see Bakhtiary and Fatemi ${ }^{7}$ ).

\section{Technique}

Although the majority of studies included specific instructions for aspects of the techniques applied, the overall explanation of the interventions was not mentioned. Three trials ${ }^{4,8,9}$ recorded that exercises were performed with correction of dynamic/static alignment of pelvis/hip/knee/foot. Studies also detailed amount of pain (two studies ${ }^{8}$ ), depth of squat or lunge (two studies ${ }^{6,16}$ ) and used biofeedback to enhance selective muscle recruitment (one study ${ }^{10}$ ).

\section{Stretching}

Stretching was included in eight of the ten trials. The majority of trials ${ }^{9-11,15,16,19}$ examined three repetitions of 30-second duration. The frequency of stretching varied as it was performed concurrently with strengthening; further details can be found in Table 2 . 


\section{Co-interventions}

Subjects participating in the exercise intervention of eight trials $^{8-11,15,16,19}$ were also receiving/allowed to receive other treatments, concurrently or as a part of that trial. Table 3 summarizes these co-interventions. The additional interventions included advice to avoid symptom-producing activity, taping, education, nonsteroidal anti-inflammatory drugs, simple analgesics, bracing, and patella mobilizations. Taping and education were the two most common co-interventions.

\section{Discussion}

This review provides evidence-based recommendations to clinicians who wish to use exercise programs to improve pain and function in patients with patellofemoral pain.

\section{Type of exercise}

The high frequency of both $\mathrm{OKC}$ and $\mathrm{CKC}$ exercises employed by the included studies indicates support for the use of both exercise types. The apparent low frequency of use of balance and proximal stability/TrA exercises questions the need to specifically train these components.

\section{Program duration}

The majority of the trials reported an intervention period of 6 weeks, except Witvrouw et al ${ }^{4}$ and Bakhtiary and Fatemi, which reported 5- and 3-week intervention periods, respectively. Therefore, an intervention period of 6 weeks could be considered the starting length for programs targeting PFPS, as the studies with intervention periods of 6 or more weeks were most commonly reported and associated with positive outcomes.

\section{Frequency and intensity}

The majority of studies (eight out of ten) prescribed 5 or more days of exercises per week. Thus, the choice of frequency is likely to be a reflection of goals of training, and

Table 3 Details of stretching and co-interventions reported

\begin{tabular}{|c|c|c|c|c|c|c|}
\hline \multirow[t]{2}{*}{ Study } & \multicolumn{4}{|c|}{ Stretching parameters } & \multicolumn{2}{|l|}{ Co-intervention } \\
\hline & Muscle/stretch & Reps & Duration & Frequency & Nonmedical & Medical \\
\hline \multirow[t]{4}{*}{ Clark et al ${ }^{15}$} & $\mathrm{H} / \mathrm{S}$ & 10 & 10 seconds & Daily & Taping & NR \\
\hline & ITB & & & & Education & \\
\hline & Quadriceps & & & & & \\
\hline & gastrocnemius & & & & & \\
\hline \multirow[t]{2}{*}{ Crossley et $\mathrm{al}^{16}$} & Sitting $H / S$ & 3 & 30 seconds & Daily & Taping (daily), & Allowed \\
\hline & Anterior hip in prone & & & & PFJ mobilization & paracetamol only \\
\hline \multirow[t]{4}{*}{ Nakagawa et al ${ }^{9}$} & Sitting $\mathrm{H} / \mathrm{S}$ & 3 & 30 seconds & $5 \times /$ week & Sitting patella & NR \\
\hline & Standing quadriceps & & & & mobilization & \\
\hline & Standing calf & & & & & \\
\hline & Standing ITB & & & & & \\
\hline Bakhtiary and & NR & & & & NR & NR \\
\hline \multicolumn{7}{|l|}{ Fatemi ${ }^{7}$} \\
\hline \multirow[t]{3}{*}{ Witvrouw et al ${ }^{19}$} & Quadriceps & 3 & 30 seconds & $3 \times /$ week & Asked not to participate & NR \\
\hline & $\mathrm{H} / \mathrm{S}$ & & & & in sport & \\
\hline & Gastrocnemius & & & & & \\
\hline Herrington and & NR & & & & Nil & Nil \\
\hline \multicolumn{7}{|l|}{ Al-Sherhi ${ }^{8}$} \\
\hline \multirow[t]{6}{*}{ Syme et $\mathrm{al}^{10}$} & Quadriceps & 3 & 30 seconds & $2 \times /$ week & Taping & Advised not \\
\hline & $\mathrm{H} / \mathrm{S}$ & & & & Education & to change \\
\hline & ITB & & & & Patellar mobilizations & current use \\
\hline & Gastrocnemius & & & & & \\
\hline & Soleus & & & & & \\
\hline & Anterior hip & & & & & \\
\hline \multirow{3}{*}{$\begin{array}{l}\text { Van Linschoten } \\
\text { et al" }\end{array}$} & Flexibility exercises & NR & NR & Daily & Education & NSAIDs $6 \%$ \\
\hline & for major thigh & & & & Brace/bandage $20 \%$ & Topical agents $3 \%$ \\
\hline & muscles, specifics NR & & & & Insoles $26 \%$ & \\
\hline \multirow[t]{4}{*}{ Song et $\mathrm{al}^{17}$} & Quadriceps & 3 & 30 seconds & $3 \times /$ week & Hot pack to quadriceps for & Nil \\
\hline & $\mathrm{H} / \mathrm{S}$ & & & & I5 minutes pre exercise, & \\
\hline & ITB & & & & cold pack to knee for 10 & \\
\hline & Calf & & & & minutes post exercise & \\
\hline \multirow[t]{3}{*}{ Kettunen et $\mathrm{al}^{23}$} & Calf & $3-5$ & 20 seconds & $2 \times /$ day & Avoidance of symptom & NR \\
\hline & $\mathrm{H} / \mathrm{S}$ & & & & producing activities & \\
\hline & Quadriceps & & & & & \\
\hline
\end{tabular}

Abbreviations: reps, repetitions; NR, not reported; H/S, hamstring; ITB, iliotibial band; NSAID, nonsteroidal anti-inflammatory drug; PFJ, patellofemoral joint. 
the need for adequate recovery following higher intensity training. Syme et al, ${ }^{10}$ for example, aimed to improve aspects of motor control such as VMO/vastus lateralis (VL) timing and dynamic alignment, and therefore may have included daily exercises of low intensity so as to reinforce motor patterns and enhance motor learning, rather than improve raw strength. Based on these results, frequency of training should be chosen with respect to the type of exercise and the perceived goals of training, and principles of overload and progression should be considered where strength is a target of intervention.

\section{Strength}

Strength as a target of treatment was explicitly stated by nine of the ten studies. The exercise interventions incorporated into protocols demonstrating positive results (knee extension, squats, stationary cycling, static quadriceps, active straight leg raise, and step up and step down exercises) contain an implicit strengthening component. Targets of strengthening exercises varied according to the trial; however, frequently included quadriceps, gluteals, hip abductors, and external rotators, and occasionally included hip adductors. Nakagawa et $\mathrm{al}^{9}$ reported significant improvement with the addition of hip and core strengthening compared with controls receiving quadriceps strengthening only. The high reporting of exercises that strengthened both hip and knee muscle groups among programs which demonstrated positive outcomes supports their inclusion in exercise programs and reflects the hip and knee strength deficits that have been shown to exist in patients with PFPS. 3,18

\section{Flexibility}

Stretching of various lower limb musculatures was included as an adjunct to exercise in eight of the ten trials. Of these, seven trials specified the stretched muscles, with all seven including hamstring and quadriceps stretches, six including gastrocnemius, five including the iliotibial band (ITB), and two including anterior hip stretches. To date only one randomized controlled trial ${ }^{20}$ has specifically investigated the effect of stretching on PFPS in isolation, concluding that although it seemed to improve flexibility and knee function there was no statistically significant improvement in pain or function with stretching alone. In a 2006 cohort study, Tyler et $\mathrm{al}^{21}$ reported that successful outcomes were correlated to demonstrated improvements in ITB and iliopsoas flexibility. The frequent inclusion of stretching in studies reporting positive outcomes further supports the use of stretching as an inclusion in exercise protocols.

\section{Selective muscle recruitment/muscle timing}

There has been a great emphasis on deficits in VMO strength and timing in interventions which selectively train this muscle. ${ }^{22}$ Fagan and Delahunt ${ }^{3}$ found that although one research study had shown improvements in VMO/VL timing with selective muscle VMO training, they did not investigate the effect of the training on symptoms. Interestingly, only two of the included studies ${ }^{10,16}$ showing positive effects of exercise incorporated selective VMO training. Syme et $\mathrm{al}^{10}$ found significant improvements with general and VMO selective strengthening compared with controls; however, there was no between-group difference. The authors ${ }^{10}$ recommend that it not be overly focused on for progressing rehabilitation.

\section{Sets and repetitions}

The review revealed a minimum of 20-40 total repetitions should be considered when prescribing exercises for patellofemoral pain.

\section{Limitations}

This review focused on exercises undertaken as part of a structured exercise program rather than general exercises (such as walking) and unstructured exercises (such as Pilates). As such, this review does not provide any insight into the role of general physical activity and unstructured exercises in the management of patients with PFPS. Like previous reviews, this review has highlighted risk of bias, due to poor methodology, in some of the primary research. One study did not report concealing of subject allocation, two did not report blinding of the primary outcome assessor, and intention to treat analysis was not reported in two of the eight studies. Co-interventions such as taping, education, and patella mobilization were also a common feature. Taping for example is reported to provide benefits independent of exercise, ${ }^{15}$ leading to opportunities for confounding. This does, however, reflect clinical practice where exercise is frequently combined with co-interventions.

\section{Conclusion \\ Implications for practice}

This systematic review builds on the current body of evidence which supports the use of exercise in reducing pain and increasing function ability in patients with PFPS. This review provides clinicians with specific parameters in order to devise an evidence-based exercise program to treat PFPS. Based on evidence from the literature, results are optimal when exercises are performed on a daily basis for 6 or more 
weeks. The interventions shown to be most effective are knee extension, squats, stationary cycling, static quadriceps, active straight leg raise, and step up and step down exercises combined with flexibility exercises. The evidence suggests that a progressive program of two to four sets of ten or more repetitions has the most benefit. These myriad of exercise options provide clinicians with the flexibility to tailor their exercise programs to suit individual needs and requirements of their patients. The findings from this review also suggest that exercise programs can be effective when used independently, or in combination with other treatments such as patella mobilization, taping, and education. This, too, provides clinicians with options as part of their management strategies, which can then be incorporated into an exercise program for patients with PFPS.

\section{Implications for research}

Exercise as a therapy for PFPS is a well-researched area. However, there are still key gaps in the literature that need to be addressed. Further research is required to determine exercise programs which are specific goal oriented to reflect patient outcomes rather than generalized programs, as is currently reported in the literature. Compliance with exercise programs are poorly reported in the literature and this could be the focus of future research, as poor compliance may lead to poor outcomes.

\section{Disclosure}

The authors report no conflicts of interest in this work.

\section{References}

1. McConnell J. The management of chondromalacia patellae: a long term solution. Aust J Physiother. 1986;32(4):215-223.

2. McConnell J. What are effective therapies for anterior knee pain. In: Wright JG, editor. Evidence Based Orthopaedics: The Best Answers To Clinical Questions. Philadelphia, PA: Saunders Elsevier Inc; 2009:634-639.

3. Fagan V, Delahunt E. Patellofemoral pain syndrome: a review on the associated neuromuscular deficits and current treatment options. $\mathrm{Br} J$ Sports Med. 2008;42(10):789-795.

4. Witvrouw E, Danneels L, van Tiggelen D, et al. Open versus closed kinetic chain exercises in patellofemoral pain: a 5-year prospective randomized study. Am J Sports Med. 2004;32(5):1122-1130.

5. Mascal C, Landel R, Powers C. Management of patellofemoral pain targeting hip, pelvis and trunk muscle function: 2 case reports. J Orthop Sports Phys Ther. 2003;33(11):647-660.

6. Heintjes E, Berger M, Bierma-Zeinstra SMA, et al. Exercise therapy for patellofemoral pain syndrome. Cochrane Database Syst Rev. 2003;(4): CD003472.
7. Bakhtiary A, Fatemi E. Open versus closed kinetic chain exercises for patellar chondromalacia. Br J Sports Med. 2008;42(2):99-102.

8. Herrington L, Al-Sherhi A. A controlled trial of weight-bearing versus non-weight-bearing exercises for patellofemoral pain. J Orthop Sports Phys Ther. 2007;37(4):155-160.

9. Nagakawa T, Muniz T, de Marche Baldon R. The effect of additional strengthening of hip abductor and lateral rotator muscles in patellofemoral pain syndrome: a randomised controlled pilot study. Clin Rehabil. 2008;22(12):1051-1060.

10. Syme G, Rowe P, Martin D, et al. Disability in patients with chronic patellofemoral pain syndrome: a randomised controlled trial of VMO selective training versus general quadriceps strengthening. Man Ther. 2009;14(3):252-263.

11. Van Linschoten R, van Middelkoop M, Berger M, et al. Supervised exercise therapy versus usual care for patellofemoral pain syndrome: an open label randomised controlled trial. BMJ. 2009;339:b4074.

12. PEDro. Physiotherapy Evidence Database. 2010. Available at: http:// www.pedro.org.au/english/downloads/pedro-scale/. Accessed 28 April, 2010.

13. Mahar CG, Sherrington C, Herbert RD, et al. Reliability of the PEDro scale for rating quality of randomised controlled trials. Phys Ther. 2003;83(8):713-721.

14. Avraham F, Aviv S, Ya'akobi P, et al. The efficacy of treatment of different intervention programs for patellofemoral pain syndrome - a single blinded randomised clinical trial. Pilot study. Scientific World J. 2007;7:1256-1262.

15. Clark D, Downing N, Mitchell J, et al. Physiotherapy for anterior knee pain: a randomised controlled trial. Ann Rheum Dis. 2000;59(9): 700-704.

16. Crossley K, Bennell K, Green S, et al. Physical therapy for patellofemoral pain. A randomised, double-blinded, placebo-controlled trial. Am J Sports Med. 2002;30(6):857-865.

17. Song CY, Lin YF, Wei TC, Lin DH, Yen TY, Jan MH. Surplus value of hip adduction in leg-press exercise in patients with patellofemoral pain syndrome: a randomised controlled trial. Phys Ther. 2009;89(5): 409-417.

18. Souza R, Powers C. Differences in hip kinematics, strength and muscle activation between subjects with and without patellofemoral pain. J Orthop Sports Phys Ther. 2009;39(1):12-19.

19. Witvrouw E, Lysens R, Bellemans J, et al. Open versus closed kinetic chain exercises for patellofemoral pain. A prospective, randomized study. Am J Sports Med. 2000;28(5):687-694.

20. Peeler J, Esther J. Effectiveness of static quadriceps stretching in individuals with patellofemoral joint pain. Clin J Sports Med. 2007;17(4): 234-241.

21. Tyler T, Nicholas S, Mullaney M, et al. The role of hip muscle function in the treatment of patellofemoral pain syndrome. Am J Sports Med. 2006;34(4):630-636.

22. Cowan S, Bennell K, Crossley K, et al. Physical therapy alters recruitment of the vasti in patellofemoral pain syndrome. Med Sci Sports Exerc. 2002;34(12):1879-1885.

23. Kettunen JA, Harilainen A, Sandelin J, et al. Knee arthroscopy and exercise only for chronic patellofemoral pain syndrome: a randomized controlled trial. BMC Med. 2007;5:38.

24. Verhagen AP, de Vet HC, de Bie RA, et al. The Delphi list: a criteria list for quality assessment of randomized clinical trials for conducting systematic reviews developed by Delphi consensus. J Clin Epidemiol. 1998;51(12):1235-1241. 


\section{Supplementary material}

The PEDro critical appraisal criteria

\section{scale ${ }^{12}$}

1. Eligibility criteria were specified

2. Subjects were randomly allocated to groups (in a crossover study, subjects were randomly allocated an order in which treatments were received)

3. Allocation was concealed

4. The groups were similar at baseline regarding the most important prognostic indicators

5. There was blinding of all subjects

6. There was blinding of all therapists who administered the therapy

7. There was blinding of all assessors who measured at least one key outcome

8. Measures of at least one key outcome were obtained from more than $85 \%$ of the subjects initially allocated to groups

9. All subjects for whom outcome measures were available received the treatment or control condition as allocated or, where this was not the case, data for at least one key outcome were analyzed by "intention to treat"

10. The results of between-group statistical comparisons are reported for at least one key outcome

11. The study provides both point measures and measures of variability for at least one key outcome

The PEDro scale is based on the Delphi list developed by Verhagen and colleagues at the Department of Epidemiology, University of Maastricht. ${ }^{24}$ The list is based on "expert consensus" not, for the most part, on empirical data. Two additional items not on the Delphi list (PEDro scale items 8 and 10) have been included in the PEDro scale. As more empirical data comes to hand it may become possible to "weight" scale items so that the PEDro score reflects the importance of individual scale items. The purpose of the PEDro scale is to help the users of the PEDro database rapidly identify which of the known or suspected randomized clinical trials (ie, RCTs or CCTs) archived on the PEDro database are likely to be valid (criteria 2-9), and could have sufficient statistical information to make their results interpretable (criteria 10-11). An additional criterion (criterion 1) that relates to the external validity (or "generalizability" or "applicability" of the trial) has been retained so that the Delphi list is complete, but this criterion will not be used to calculate the PEDro score reported on the PEDro web site. The PEDro scale should not be used as a measure of the "validity" of a study's conclusions. In particular, we caution users of the PEDro scale that studies which show significant treatment effects and which score highly on the PEDro scale do not necessarily provide evidence that the treatment is clinically useful. Additional considerations include whether the treatment effect was big enough to be clinically worthwhile, whether the positive effects of the treatment outweigh its negative effects, and the cost-effectiveness of the treatment. The scale should not be used to compare the "quality" of trials performed in different areas of therapy, primarily because it is not possible to satisfy all scale items in some areas of physiotherapy practice.

Last amended June 21, 1999
Journal of Multidisciplinary Healthcare

\section{Publish your work in this journal}

The Journal of Multidisciplinary Healthcare is an international, peerreviewed open-access journal that aims to represent and publish research in healthcare areas delivered by practitioners of different disciplines. This includes studies and reviews conducted by multidisciplinary teams as well as research which evaluates the results or conduct of such teams or healthcare processes in general. The journal covers a wide range of areas and welcomes submission from practitioners at all levels, from all over the world. The manuscript management system is completely online and includes a very quick and fair peer-review system. Visit http://www.dovepress.com/testimonials.php to read real quotes from published authors. 